\title{
Galactic substructure and dark-matter annihilation in the Milky Way halo
}

\author{
Marc Kamionkowski, ${ }^{1, *}$ Savvas M. Koushiappas, ${ }^{2, \dagger}$ and Michael Kuhlen ${ }^{3, \$}$ \\ ${ }^{1}$ California Institute of Technology, Mail Code 350-17, Pasadena, California 91125, USA \\ ${ }^{2}$ Department of Physics, Brown University, 182 Hope Street, Providence, Rhode Island 02912, USA \\ ${ }^{3}$ Theoretical Astrophysics Center, University of California Berkeley, Berkeley, California 94720, USA
}

(Received 19 January 2010; published 22 February 2010)

\begin{abstract}
We study the effects of substructure on the rate of dark-matter annihilation in the Galactic halo. We use an analytic model for substructure that can extend numerical simulation results to scales too small to be resolved by the simulations. We first calibrate the analytic model to numerical simulations, and then determine the annihilation boost factor, for standard weakly interacting massive particle (WIMP) models as well as those with Sommerfeld (or other) enhancements, as a function of galactocentric radius in the Milky Way. We provide an estimate of the dependence of the gamma-ray intensity of WIMP annihilation as a function of angular distance from the Galactic center. This methodology, coupled with future numerical simulation results can be a powerful tool that can be used to constrain WIMP properties using Fermi all-sky data.
\end{abstract}

DOI: 10.1103/PhysRevD.81.043532

PACS numbers: 95.35.+d, 98.35.Gi, 98.35.Pr, 98.62.Gq

\section{INTRODUCTION}

Weakly interacting massive particles (WIMPs) provide perhaps the most promising class of dark-matter candidates. These are particles that arise in theories of new electroweak-scale physics, such as low-energy supersymmetry [1] or models with universal extra dimensions [2]. Experiments that seek to directly detect these particles in low-background experiments, or to indirectly detect them through observation of energetic neutrinos from WIMP annihilation in the Sun, are now beginning to dig into the favored WIMP parameter space. However, there are also prospects for indirectly detecting WIMPs through observation of gamma rays and/or cosmic-ray positrons, antiprotons, or antideuterons from WIMP annihilation in the Galactic halo. These annihilation products have received considerable attention in the recent literature with the attribution of some reported (and still controversial) cosmic-ray anomalies [3] to WIMP-annihilation products.

The total rate at which WIMPs annihilate in the Galactic halo is proportional to the volume integral of the square of the dark-matter density. In the canonical (and simplest) model, the dark matter is smoothly distributed in the halo in a spherically symmetric way with a dark-matter density $\rho(r)$ that is a monotonically decreasing function of $r$; for example, it is often modeled as an isothermal or a NavarroFrenk-White (NFW) profile [4].

However, analytic arguments and numerical simulations show that there should be substructure in the dark-matter distribution in the Galactic halo [5-7]. The dark matter may be clumped; some of it may be bound in higherdensity self-bound subhalos; and some may be in tidal

\footnotetext{
*kamion@tapir.caltech.edu

†koushiappas@brown.edu

mqk@astro.berkeley.edu
}

streams [8]. This is an outcome of hierarchical clustering, in which small dense halos form first and then merge to form progressively larger structures. If dark matter in the halo is clumped, then the total annihilation rate will be enhanced by some boost factor [9], the increased rate per unit volume in dense regions outweighing the decreased rate per unit volume in lower-density regions.

In the canonical WIMP scenario, the substructure may have a roughly scale-invariant distribution in substructure mass/size extending all the way down to substructures on mass scales $\sim 10^{-10} M_{\odot}[7,10,11]$, roughly 22 orders of magnitude smaller than the $\sim 10^{12} M_{\odot}$ Milky Way halo. While state-of-the-art numerical calculations now have the resolution to simulate several decades in this hierarchy, they are very far from being able to follow the survival and evolution of the smallest substructures through all of the generations in the structure-formation hierarchy that result in a Milky Way halo. Analytic calculations of these survival probabilities are difficult $[12,13]$.

However, given that the smallest subhalos are likely to be the densest, the boost factor may depend significantly on the existence of these substructures. This is particularly true in models with a Sommerfeld enhancement in the annihilation rate [14] - those where the annihilation rate increases with lower WIMP velocities-since the smallest subhalos are also likely to have the smallest velocity dispersion.

In a previous paper [15], we presented an analytic model to describe the self-similar substructure expected from hierarchical clustering. The model predicted a high-density power-law tail for the probability for a given point in the halo to be in a clump of density $\rho$. Subsequent to that paper, another appeared [16] that presented $N$-body simulations that showed this power-law tail. After calibration to numerical simulations, the model can be used to extrapolate the results of numerical simulations to substructure- 
mass scales far smaller than those currently accessible to the simulations. We investigated in Ref. [15] the dependence of the boost factor assuming a canonical WIMPannihilation rate (i.e., no Sommerfeld enhancement). A complementary analytical approach to this problem, based on the stable clustering hypothesis, was recently presented by [17].

In this paper we revisit and extend those calculations. We first describe in Sec. II the analytic model. We extend the model by including a finite width for the smoothly distributed component of dark matter in addition to the high-density power-law tail. We then use in Sec. III stateof-the-art $N$-body simulations to measure the width of the smooth component and the amplitude of the power-law tail and thus calibrate the analytic model to current simulations. We moreover determine how the substructure distribution varies with galactocentric radius in the halo. In Sec. IV we use the calibrated model to determine the boost factors for canonical WIMPs and for WIMPs with a Sommerfeld enhancement to the annihilation rate. Section V reviews the substructure-model parameters expected from WIMP models, and Sec. VI determines the angular dependence of the intensity of gamma-ray radiation both with and without the substructure boost factor we obtain. Section VII reviews the model and results and then provides some comments, caveats, and directions for future development of the model.

\section{SUBSTRUCTURE MODEL}

Before discussing our analytic model for substructure we begin by presenting the canonical smooth-halo model against which the substructure model will be compared. We take as the canonical model for the Galactic halo an NFW profile,

$$
\bar{\rho}(r)=\frac{4 \rho_{s}}{\left(r / r_{s}\right)\left(1+r / r_{s}\right)^{2}},
$$

as a function of galactocentric radius $r$, with parameters $\rho_{s}=0.051 \mathrm{GeV} \mathrm{cm}^{-3}$ and $r_{s}=21.7 \mathrm{kpc}$ taken to provide a reasonable fit to the Milky Way rotation curve.

If dark matter in the halo is clumped, then the densities at all points with the same $r$ will not necessarily be the same. Instead, there will be some probability distribution function $P(\rho, r)$ defined so that $P(\rho, r) d \rho$ is the probability that a particular point in the Galactic halo (at some fixed galactocentric radius $r$ ) has a density between $\rho$ and $\rho+$ $d \rho$. According to the arguments of Ref. [15], a fraction $f_{s}$ of the volume of the halo (in fact most of the volume; $f_{s} \simeq$ 1 , as we will see) should be filled with a smooth darkmatter component with density $\rho_{h}$, and a fraction $1-$ $f_{s} \ll 1$ will consist of a high-density clumped component, with something like a power-law distribution of densities. Thus, the probability distribution function that we use is

$$
\begin{aligned}
P(\rho ; r)= & \frac{f_{s}}{\sqrt{2 \pi \Delta^{2}}} \frac{1}{\rho} \exp \left\{-\frac{1}{2 \Delta^{2}}\left[\ln \left(\frac{\rho}{\rho_{h}} e^{\Delta^{2} / 2}\right)\right]^{2}\right\} \\
& +\left(1-f_{s}\right) \frac{1+\alpha(r)}{\rho_{h}} \Theta\left(\rho-\rho_{h}\right)\left(\frac{\rho}{\rho_{h}}\right)^{-(2+\alpha)},
\end{aligned}
$$

where $\Theta(x)$ is the Heaviside step function. Here, the first term describes the smooth host halo component as having a Gaussian distribution in $\ln \rho$ with mean density $\rho_{h}$ and a Gaussian width in $\ln \rho$ of $\Delta$. This distribution peaks at a density $\rho_{0}=e^{-3 \Delta^{2} / 2} \rho_{h}$, a density slightly below $\rho_{h}$.

The second term in Eq. (2) is the high-density power-law tail due to substructures that remain from earlier generations in the structure-formation hierarchy. The parameter $\rho_{h}$ in Eq. (2) is in fact a function of $r$; we will see below that $\rho_{h}(r) \simeq \bar{\rho}$ to very good accuracy. There may also be further $r$ dependence in $P(\rho ; r)$ through an $r$ dependence of the parameters $\Delta, \alpha$, and $f_{s}$. As we will see, our simulations are not yet good enough to allow the $r$ dependence of $\alpha$ to be resolved, and so we take it to be a constant. The simulations are, however, sufficiently resolved to see a strong dependence of $f_{s}(r)$ on $r$, which we detail below.

The distribution $P(\rho ; r)$ in Eq. (2) is normalized so that $\int_{0}^{\infty} P(\rho ; r) d \rho=1$. It can be integrated to give the mean density,

$$
\begin{aligned}
\bar{\rho}(r) & =\int_{0}^{\rho_{\max }} \rho P(\rho) d \rho \\
& =f_{s} \rho_{h}+\left(1-f_{s}\right) \rho_{h} \begin{cases}\frac{1+\alpha}{\alpha}\left[1-\left(\frac{\rho_{\max }}{\rho_{h}}\right)^{-\alpha}\right] ; & \alpha \neq 0 \\
\left(1-f_{s}\right) \rho_{h} \ln \frac{\rho_{\max }}{\rho_{h}} ; & \alpha=0 .\end{cases}
\end{aligned}
$$

This thus provides a relation between the mean density $\rho_{h}(r)$ of the smooth component and the canonical host halo density $\bar{\rho}$. Strictly speaking, this relation requires the maximum density $\rho_{\max }$ out to which the power-law tail extends. However, we will see that numerically $1-f_{s}$ is so small that $\rho_{h}(r) \simeq \bar{\rho}(r)$ is a very good approximation for practical purposes.

\section{CALIBRATION TO SIMULATIONS}

We make use of the Milky Way-like halo in the Via Lactea II simulation [18] and calibrate our model to the $P(\rho ; r)$ measured from the simulated particle distribution. Figure 1 shows the PDF $P(\rho)$ derived from Via Lactea II for three galactocentric radii, at $10 \mathrm{kpc}$ (lowest solid line, red), at $100 \mathrm{kpc}$ (middle solid line, blue), and at $300 \mathrm{kpc}$ (highest solid line, cyan). To obtain this $P(\rho)$, we consider an ellipsoidal shell that follows an isodensity contour in the halo. For each particle in that shell, we calculate $\delta_{i}=\rho / \bar{\rho}$ where $\rho$ is estimated in the usual manner from the nearest $N$ neighbors using a symmetric smoothed particle hydrodynamics kernel and the median density $\bar{\rho}$ is obtained from all the particles in that ellipsoidal shell. These $\delta_{i}$ are then binned in equally spaced bins in $\log _{10}(\delta)$. In each of these bins, we calculate $P\left(\log _{10} \delta\right)=\sum_{i} \delta_{i}^{-1}$ where the sum is 


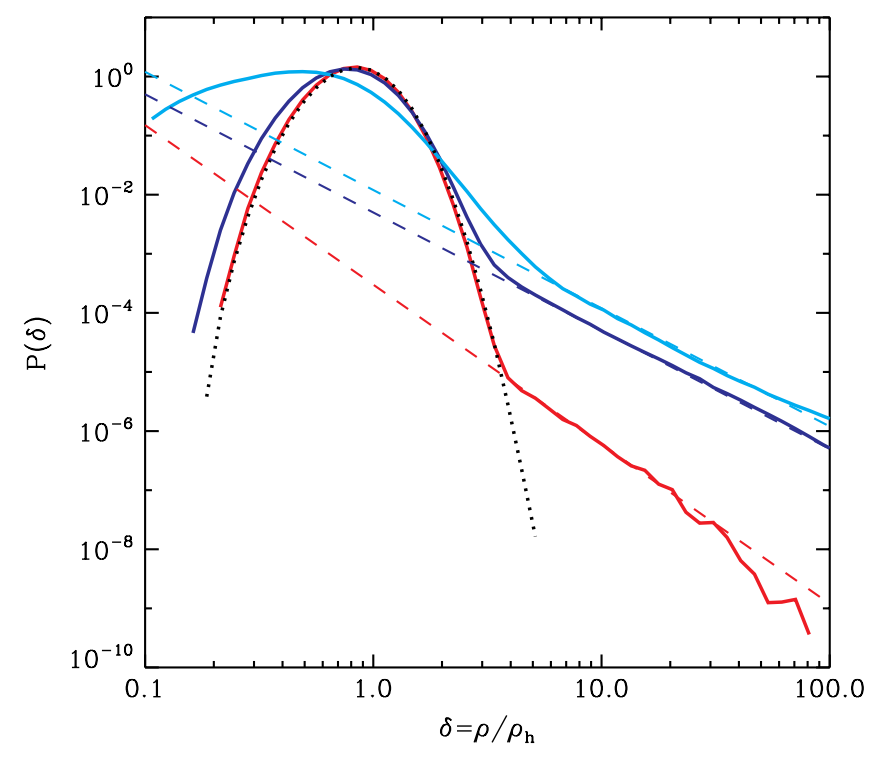

FIG. 1 (color online). The probability distribution function $P(\delta)$ obtained from simulations. The solid curves are the simulation results at $r=300,100$, and $10 \mathrm{kpc}$ (from top to bottom). The dashed curves show our analytic approximations to the power-law tail. The dotted curve indicates the contribution to the finite width of the smooth component at $10 \mathrm{kpc}$ from Poisson fluctuations due to the use of $N=32$ neighbors in the density estimator. Note that the mean host halo density $\rho_{h}$ to which the $x$ axis is normalized is $\sim 175 \times(\sim 4900 \times)$ smaller for the 100 kpc $(300-\mathrm{kpc})$ curve than in the 10-kpc curve.

over all particles in that bin; the $\delta_{i}^{-1}$ weighting gives a volume-fraction distribution. The distribution in $\log _{10} \delta$ is then converted to a distribution in $\delta$ and normalized.

\section{A. Power-law tail}

The central features of Fig. 1 relevant here are the highdensity power-law tails predicted by Ref. [15] (and seen already in simulations [16]). The figure shows that the amplitude of the high-density power-law tail is larger at larger radii. This can be attributed largely to the fact that the mean density $\bar{\rho}$ is $\sim 175$ times lower at $100 \mathrm{kpc}$ than at $10 \mathrm{kpc}$, and another factor $\sim 30$ times lower at $300 \mathrm{kpc}$, and so the ratio of the density in substructures to the mean density is higher at larger radii.

We now use this simulation to calibrate the analytic model at a variety of radii $r$, from 4 to $300 \mathrm{kpc}$. At each radius we fit for the power-law parameters $\alpha$ and $f_{s}$. We find that at radii greater than $\sim 20 \mathrm{kpc}$, the smooth-halo fraction is well approximated by

$$
1-f_{s}(r)=7 \times 10^{-3}\left(\frac{\bar{\rho}(r)}{\bar{\rho}(r=100 \mathrm{kpc})}\right)^{-0.26} .
$$

Note that at radii less than $\sim 20 \mathrm{kpc}, 1-f_{s}(r)$ drops faster than Eq. (4); for example, $1-f_{s}(10 \mathrm{kpc})=4 \times 10^{-4} \approx$ $1.5 \times 10^{-3}(\bar{\rho}(10 \mathrm{kpc}) / \bar{\rho}(100 \mathrm{kpc}))^{-0.26}$. This close to the center, however, the clumpiness of the simulated halo is

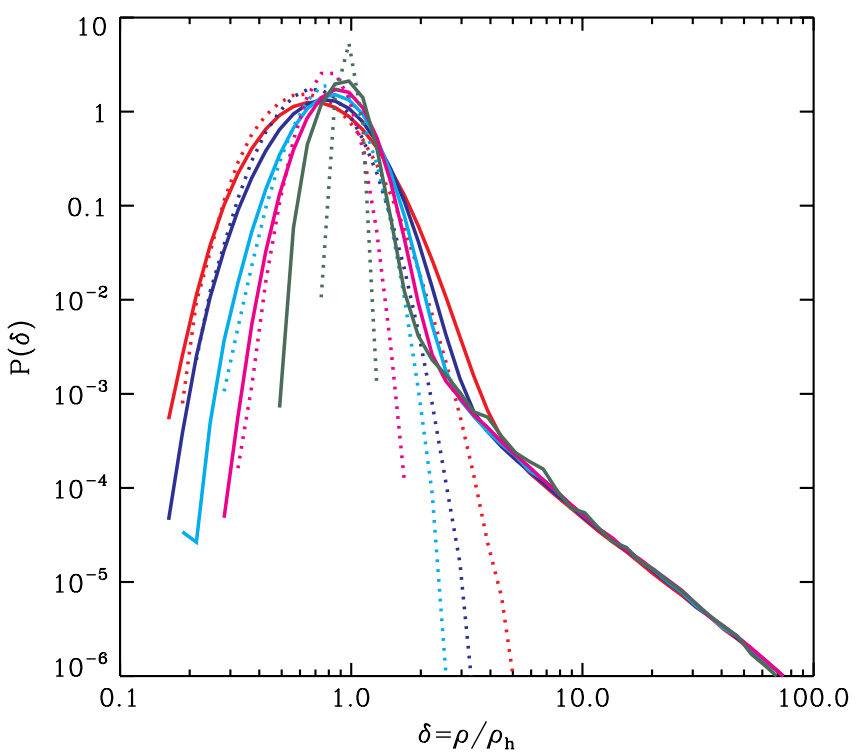

FIG. 2 (color online). The probability distribution function $P(\delta)$ at $100 \mathrm{kpc}$ for particle densities estimated from the nearest $N=(16,32,64,128,1024)$ neighbors.

likely artificially suppressed due to finite resolution effects. The best-fit values of $\alpha$ are $0.0 \pm 0.1$ at all radii greater than $20 \mathrm{kpc}$. In the following, we implicitly assume $\alpha=0$ and the radial dependence in $f_{s}$ given by Eq. (4).

\section{B. Finite width of the smooth component}

The simulation results shown in Fig. 1 show a finite width $\Delta$ for the smooth component. However, care must be taken as Poisson fluctuations due to the finite number $N$ of nearest neighbors in the density estimator will also contribute to the width. In Fig. 2 we show $P(\delta)$ at $100 \mathrm{kpc}$ for densities determined with $N=16,32,64$, 128, and 1024. The dotted curves indicate the expected contribution to the width from Poisson fluctuations (and note that the true and Poisson widths should add in quadrature), which we obtained by running the density estimator on a randomly distributed sample of $10^{6}$ particles. As $N$ is increased, the width of the smooth component decreases, but not quite as fast as the Poisson fluctuations, and by $N=$ 1024 it is clear that the true width has been resolved to be about $\Delta \simeq 0.2$. At $10 \mathrm{kpc}$ (not shown here) it remains unresolved, and we conclude only that $\Delta \lesssim 0.2$ at radii less than $100 \mathrm{kpc}$.

\section{ANNIHILATION BOOST FACTOR}

We now consider the boost of the annihilation rate in a halo with substructure relative to the rate in the canonical smooth-halo model.

The annihilation rate (per unit volume) at any point in the Galactic halo is

$$
\Gamma=\langle\sigma v\rangle \frac{\rho^{2}}{2 m_{\chi}^{2}},
$$


where $\langle\sigma v\rangle$ is the annihilation cross section (times relative velocity $v$, averaged over the velocity distribution of the halo), and $m_{\chi}$ is the WIMP mass; i.e., $\langle\sigma v\rangle=$ $\int_{0}^{\infty} d v f(v) \sigma v$, where $f(v)$ [normalized to $\int_{0}^{\infty} f(v) d v=$ 1] is the WIMP pairwise velocity distribution at that point in the Galactic halo. For the canonical WIMP, $\sigma v$ is approximately velocity independent at Galactic-halo velocities, and so $\langle\sigma v\rangle=(\sigma v)$, a constant. If $\sigma v$ depends on velocity, then $\langle\sigma v\rangle$ may vary from one point in the halo to another due to the possible variation of $f(v)$ with position in the halo.

\section{A. Standard velocity dependence}

Let us first consider the canonical WIMP where the cross-section factor $\langle\sigma v\rangle$ is velocity independent. In this case, the change in the velocity dispersion that may accompany clumping is irrelevant. The total annihilation rate in some volume $V$ is then proportional to a volume integral of the density squared-i.e., $\propto \int \rho^{2} d V$. The annihilation rate for a fixed total mass of dark-matter enclosed within a given volume will thus be enhanced by a "boost-factor" if the matter is not uniformly distributed within that volume. For the canonical WIMP, this boost factor $B(r)$ is the ratio

$$
B(r)=\frac{\int \rho^{2} d V}{\int[\bar{\rho}(r)]^{2} d V}=\int_{0}^{\rho_{\max }} P(\rho, r) \frac{\rho^{2}}{[\bar{\rho}(r)]^{2}} d \rho,
$$

where the second equality follows since $P(\rho, r)=$ $(1 / V)(d V / d \rho)$ (with $V$ the volume in the halo). The quantity $\rho_{\max }$ is the maximum density. We take that to be $\sim 80 \mathrm{GeV} \mathrm{cm}^{-3}$, which is about 5 times the mean virial density of the rare first collapsed structures in the Universe (178 times the mean density of the Universe at $z \approx 40$ ); we will say more later about $\rho_{\max }$.

In the standard WIMP model, where $\langle\sigma v\rangle=$ constant, the boost factor $B(r)$ is given by (noting that $\bar{\rho} \simeq \rho_{h}$ )

$$
B(r)=f_{s} e^{\Delta^{2}}+\left(1-f_{s}\right) \frac{1+\alpha}{1-\alpha}\left[\left(\frac{\rho_{\max }}{\rho_{h}}\right)^{1-\alpha}-1\right] .
$$

There is $r$ dependence in this boost factor via Eq. (4) in $f_{s}$, via Eq. (1) in $\rho_{h} \approx \bar{\rho}$, and in principle also in $\Delta$, although it turns out to be negligible.

There are two contributions to this boost factor: The first comes from the finite width $\Delta$ of the smooth componenti.e., $B_{s}=f_{s} e^{\Delta^{2}}$-and it depends very strongly on $\Delta$. Given that we find $\Delta \lesssim 0.2$ in our simulations, we infer that the boost factor due to the finite width of the smooth component is no more than a few percent.

We now turn to the second term, that due to substructure, the central focus of this work. Given that $\rho_{\max } \gg \bar{\rho}$ in the Milky Way halo, the boost factor would be essentially independent of $\rho_{\max }$ if $\alpha$ were $\alpha>1$. However, we find in the simulations that $\alpha<1$ in a Milky Way-like halo, in which case the boost-factor results for the canonical WIMP do indeed depend on $\rho_{\max }$. The fact that the integral in
Eq. (6) is dominated by the high-density end implies that the boost factor due to clumping is determined primarily by the early-collapsing highest-density (and lowest-mass) substructures.

What is an appropriate value for $\rho_{\max }$ ? A first guess might be the virial density of the earliest-collapsing halos, $\rho_{\text {vir }}=178 \rho_{\text {crit }}\left(z_{c}\right) \simeq 16 \mathrm{GeV} \mathrm{cm}^{-3}\left(z_{c} / 40\right)^{3}$ (for a collapse redshift $z_{c} \gg 0$ ). However, depending on its density profile, most of a halo's volume might have considerably higher densities. In Fig. 3 we show the density probability distribution $P(\rho) \equiv 1 / V d V / d \rho$ for an isolated NFW halo. At low densities, in the outskirts of the halo where $\rho \sim r^{-3}$, the density probability falls as $\rho^{-2}$, matching the powerlaw tail of the local density probability function [Eq. (2)]. In the innermost regions of the halo, where $\rho \sim r^{-1}$, we have $P(\rho) \sim \rho^{-4}$. The transition between these two regimes occurs at $\rho_{s}=1 / 12 c^{3} / f(c) \rho_{\text {vir }}$, where $c=$ $R_{\text {vir }} / r_{s}$ is the concentration of the halo and $f(c)=\ln (1+$ $c)-c /(1+c)$. We set $\rho_{\max }=\rho_{s}=1 / 12 c^{3} / f(c) \rho_{\text {vir }}$, which then depends only on the concentration with which the earliest-collapsing halos are born. Numerical simulations [7] indicate low natal concentrations of $c \approx 2-5$, corresponding to $\rho_{\max } \approx 1.5-11 \rho_{\text {vir }}$. For definiteness, we pick an intermediate value of $c=3.5$ and $z_{c}=40$, giving $\rho_{\text {max }}=80 \mathrm{GeV} \mathrm{cm}^{-3}$.

Close to the center, the clumped fraction $1-f_{s}$, as determined from the numerical simulation, is so small that the boost factor remains close to unity. As the mean halo density $\rho_{h}$ decreases with radius while the clumped fraction increases [cf. Eq. (4)], the local boost factor grows considerably in the outer regions of the halo. Note, how-

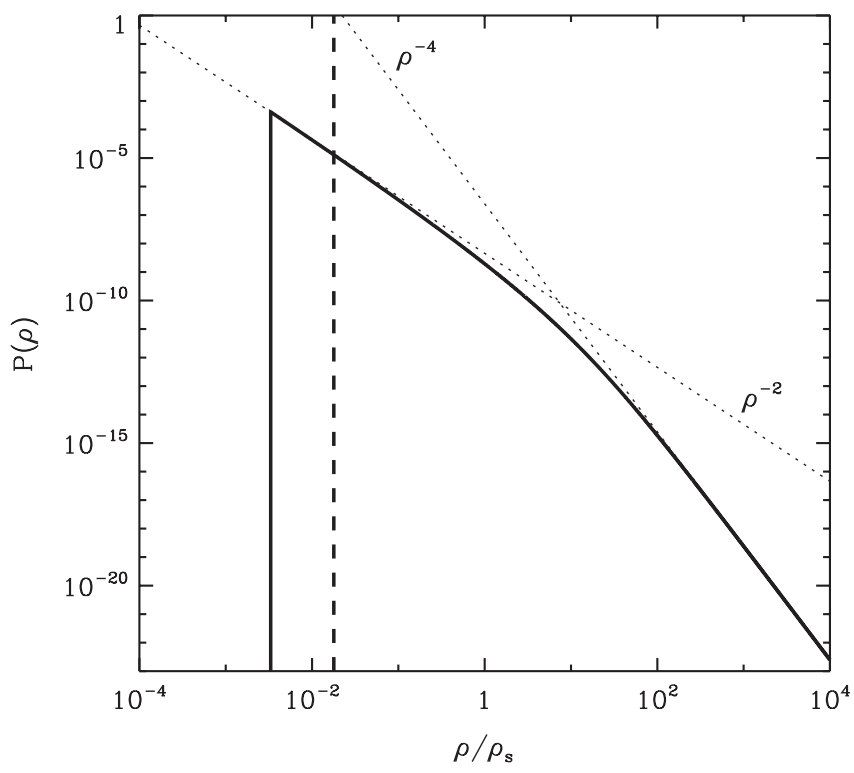

FIG. 3. The density probability distribution function $P(\rho)$ for an isolated NFW halo with concentration $c=10$, truncated at $r_{\text {vir }}$. The $P(\rho) \sim \rho^{-2}$ behavior continues beyond $\rho_{\text {vir }}$ (vertical dashed line) to $\sim \rho\left(r_{s}\right)=1 / 12 c^{3} / f(c) \rho_{\text {vir }}$. 
ever, that the total luminosity of the halo does not increase in proportion to this local boost factor. The overall luminosity is dominated by radii $\lesssim r_{s}$, and the total boost from substructure within a radius $R$ must be evaluated numerically,

$$
B(<R)=\frac{\int_{0}^{R} B(r) \rho(r)^{2} r^{2} d r}{\int_{0}^{R} \rho(r)^{2} r^{2} d r}
$$

We show in Fig. 4 the differential and cumulative luminosity boost factor as a function of radius for the Via Lactea II host halo, assuming $\alpha=0$ and $1-f_{s}(r)$ as given in Eq. (4). The boost factor remains close to unity in the center and only reaches 1.5 at the Sun's distance of $8 \mathrm{kpc},{ }^{1}$ implying that if the WIMP-annihilation cross section has the canonical $\langle\sigma v\rangle \propto$ constant dependence on the velocity, the local boost from substructure is unlikely to provide the missing factor of 100-1000 needed to explain the cosmicray anomalies (see also [19]). The total luminosity of the halo, however, can be appreciably boosted by substructure. The cumulative boost $B(<r)$ increases to $\sim 17$ at the virial radius.

Before proceeding further, we note that if $\alpha=0$ (and $\left.\rho_{\max } \gg \bar{\rho}\right)$, then the boost factor is $B(r)=f_{s}+(1-$ $\left.f_{s}\right)\left(\rho_{\max } / \bar{\rho}\right)$, an expression that is easily understood. The first term is simply the usual annihilation rate due to the smoothly distributed dark matter. The second is that due to clumping. If the integrand in Eq. (6) is dominated by the high-density end, it implies that most of the annihilation in the clumped component is taking place in the smallest and densest subhalos. If so, then the annihilation rate, per unit volume, from substructure should be proportional simply to the spatial density of the subhalos (i.e., how many there are per unit volume), which itself should be proportional to the ratio of subhalo to host halo density $\left(\rho_{\max } / \bar{\rho}\right)$; this is consistent with Eq. (7) if $\alpha=0$.

\section{B. Sommerfeld enhancement}

Suppose now that the annihilation cross section is such that the thermally averaged cross section is $\sigma v \propto \bar{v}^{-\beta}$, where $\bar{v}$ is the rms relative velocity for annihilating WIMPs. There will then be an additional enhancement in the annihilation rate since lower-mass subhalos will have smaller velocity dispersions. We account for this additional effect as follows: We first recall that the characteristic density of a first-generation halo collapsing at $z=40$ with a concentration of $c=3.5$ is $\rho_{s}=80 \mathrm{GeV} \mathrm{cm}^{-3}$, and that the corresponding characteristic velocity is $v_{s} \equiv$ $\sqrt{G M\left(<r_{s}\right) / r_{s}} \simeq 1.0 \times 10^{-3} \mathrm{~km} \mathrm{sec}^{-1}$. We then note that a typical Milky Way host halo of mass $2 \times 10^{12} M_{\odot}$ and concentration $c=15$ at $z=0$ has a corresponding char-

\footnotetext{
${ }^{1}$ It would be only 1.14 using the value of $1-f_{s}$ measured at $10 \mathrm{kpc}$, instead of Eq. (4).
}

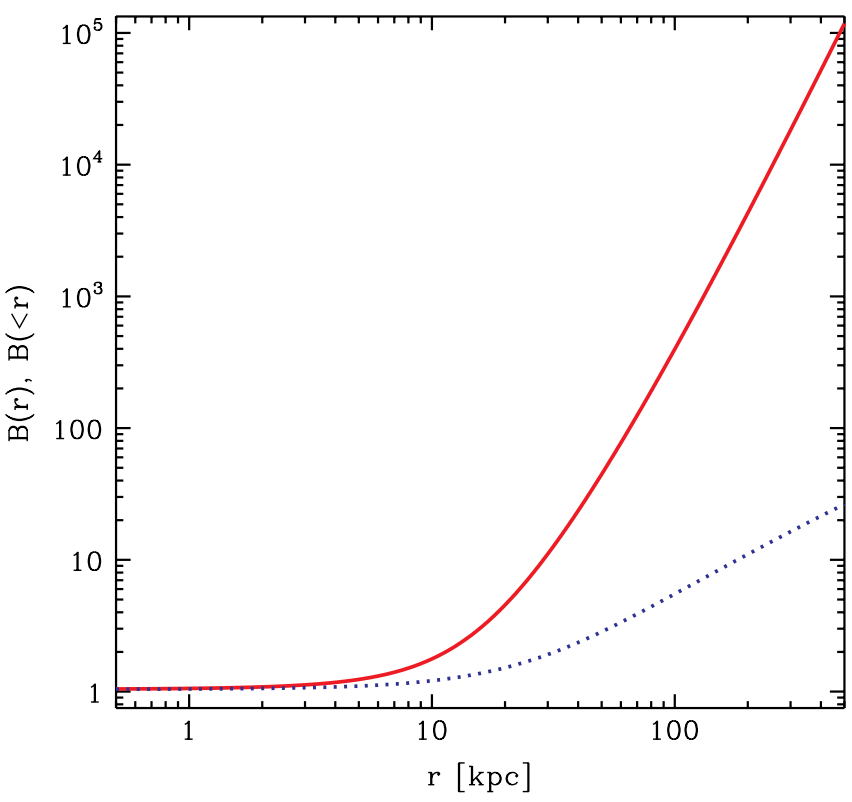

FIG. 4 (color online). The local substructure boost $B(r)$ (solid line) and the cumulative luminosity boost $B(<r)$ (dotted line), as a function of radius.

acteristic density and velocity of $\rho_{s}=0.076 \mathrm{GeV} \mathrm{cm}^{-3}$ and $v_{s}=200 \mathrm{~km} \mathrm{sec}^{-1}$. This thus suggests a rough scaling $v \propto \rho^{-1.75}$. We emphasize that this scaling is only meant to very roughly capture the relation between density and relative velocity of the dark-matter particles. In reality there likely is no such simple one-to-one relationship between these two quantities, since regions with similar densities can be bound to subhalos of very different masses and hence have very different velocity dispersions. However, as will become clear below, our results are not very sensitive to the exact value of the power-law exponent, as long as regions of higher density (at a fixed galactocentric distance) typically have lower velocity dispersions.

In the following, we consider only the Sommerfeldenhanced boost factor from the clumped component and disregard the small contribution from the finite width of the smooth component. We further assume that the dark-matter velocity dispersion $\bar{v}_{\mathrm{MW}} \simeq 220 \mathrm{~km} \mathrm{sec}^{-1}$ in the Galactic halo does not vary with galactocentric radius $r$. Strictly speaking, this constancy does not hold for a selfgravitating NFW distribution. Realistically, though, the Milky Way disk contributes very significantly to the potential in the inner Galaxy, and so the dark-matter spatial/ velocity distribution in the inner Galaxy cannot be a pure self-gravitating NFW distribution. Our assumption of a constant dark-matter velocity dispersion is probably closer to the truth than the radial change in the velocity dispersion implied by an NFW distribution.

With these approximations and assumptions, the boost factor is 


$$
B(r)=f_{s}+\left(1-f_{s}\right)(1+\alpha) \int_{\bar{\rho}}^{\rho_{\max }} \frac{d \rho}{\bar{\rho}}\left(\frac{\rho}{\bar{\rho}}\right)^{-\alpha}\left(\frac{v_{\mathrm{MW}}}{v(\rho)}\right)^{\beta},
$$

which we then integrate with the relation $v(\rho)=$ $v_{0}\left(\rho / \rho_{\max }\right)^{-1.75}$ to find

$$
\begin{aligned}
B(r)= & f_{s}+\left(1-f_{s}\right) \frac{1+\alpha}{1+1.75 \beta-\alpha}\left(\frac{v_{\mathrm{MW}}}{v_{0}}\right)^{\beta} \\
& \times\left[\left(\frac{\rho_{\max }}{\bar{\rho}}\right)^{1-\alpha}-\left(\frac{\rho_{\max }}{\bar{\rho}}\right)^{-1.75 \beta}\right] .
\end{aligned}
$$

Again, for the values of $\alpha$ we see in the simulation, the integrand in Eq. (9) is dominated by the high end, increasingly so for $\beta>0$. Now, even though $1-f_{s}$ may be small (the clumped fraction is small), the velocity enhancement $\left(v_{\mathrm{MW}} / v_{0}\right)^{\beta}$ may be large, even for values $\beta \simeq 1$. Again, if the integrand is dominated by the high-density tail, it implies that most of the annihilation in the clumped fraction is occurring in the lowest-mass highest-density regions. And if so, then the annihilation rate per unit volume should again be proportional simply to the ratio of subhalo to host halo density $\left(\rho_{\max } / \bar{\rho}\right)$, which is again implied in Eq. (10) if $\alpha=0$.

If the mass of the force carrier particle mediating the annihilation is nonzero, the Sommerfeld effect saturates at a finite velocity $v_{\text {sat }}$, when the de Broglie wavelength of the particle becomes longer than the range of interaction. With such a saturation the integral in Eq. (9) is split into two parts: one from $\bar{\rho}$ to $\rho_{\text {sat }}=\rho_{\text {max }}\left(v_{0} / v_{\text {sat }}\right)^{1 / 1.75}$ with a velocity-dependent Sommerfeld enhancement term $\left(v_{\mathrm{MW}} / v(\rho)\right)^{\beta}$, and a second part from $\rho_{\text {sat }}$ to $\rho_{\max }$ with a

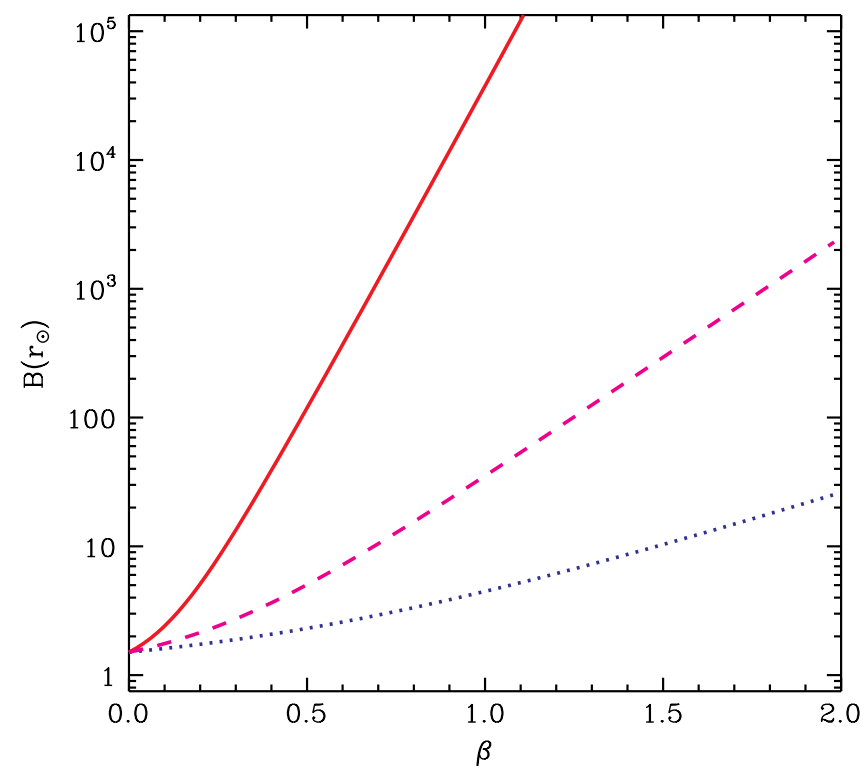

FIG. 5 (color online). The boost factor at the solar radius as a function of the parameter $\beta$, for no saturation (solid line), $v_{\text {sat }}=$ $10^{-4} c$ (dotted line), and $v_{\text {sat }}=10^{-5} c$ (dashed line). The Sommerfeld-like enhancement grows as $v^{-\beta}$ until $v_{\text {sat }}$. constant enhancement factor of $\left(v_{\mathrm{MW}} / v_{\mathrm{sat}}\right)^{\beta}$,

$$
\begin{aligned}
B(r)= & f_{s}+\left(1-f_{s}\right)(1+\alpha)\left(\frac{v_{\mathrm{MW}}}{v_{\mathrm{sat}}}\right)^{\beta}\left[\frac{1}{1+1.75 \beta-\alpha}\right. \\
& \times\left(\left(\frac{\rho_{\mathrm{sat}}}{\bar{\rho}}\right)^{1-\alpha}-\left(\frac{\rho_{\mathrm{sat}}}{\bar{\rho}}\right)^{-1.75 \beta}\right) \\
& \left.+\frac{1}{1-\alpha}\left(\left(\frac{\rho_{\mathrm{max}}}{\bar{\rho}}\right)^{1-\alpha}-\left(\frac{\rho_{\mathrm{sat}}}{\bar{\rho}}\right)^{1-\alpha}\right)\right]
\end{aligned}
$$

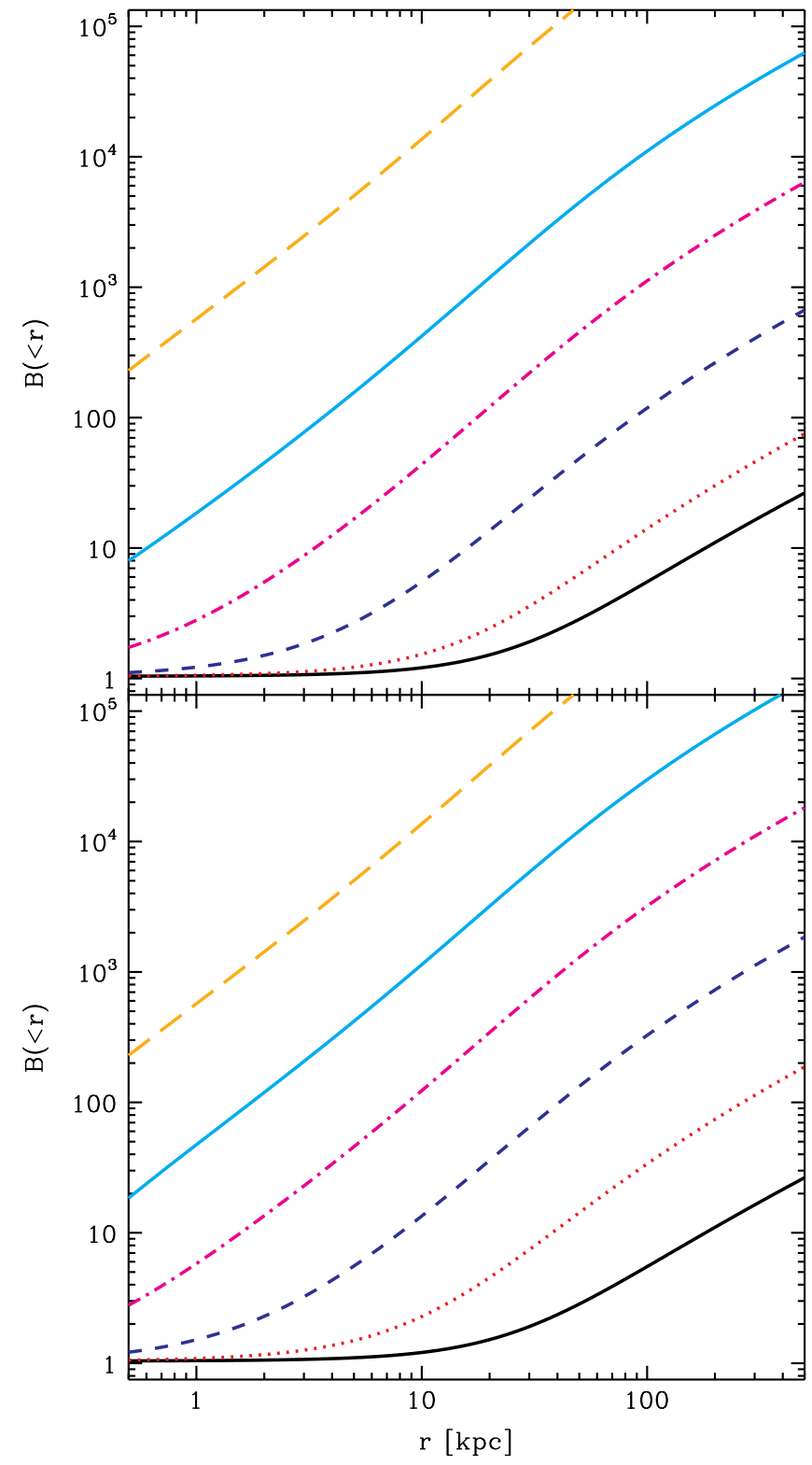

FIG. 6 (color online). The cumulative boost factor as a function of distance from the Galactic center. In both panels the solid curve represents the canonical case $(\beta=0)$. Top panel: $B(<r)$ for different values of $\beta$ with no velocity saturation: $\beta=0.1$, $0.3,0.5,0.7$, and 1.0 from bottom to top. Bottom panel: $B(<r)$ for $\beta=1.0$ and different values of the saturation velocity: $v_{\text {sat }} / c=10^{-4}, 10^{-5}, 10^{-6}, 10^{-7}$, and 0 from bottom to top. 
Figure 5 shows the boost factor at the solar radius as a function of the velocity parameter $\beta$. The solid line depicts the case without saturation, and the dotted and dashed lines with $v_{\text {sat }} / c=10^{-4}$ and $10^{-5}$, respectively. We use $\rho_{\max }=$ $80 \mathrm{GeV} \mathrm{cm}^{-3}$ and $v_{0}=1.0 \times 10^{-3} \mathrm{~km} \mathrm{sec}^{-1}$ here. With Sommerfeld enhancement it is possible to get very large substructure boost factors even at the solar radius. This substructure boost, of course, applies in addition to the Sommerfeld enhancement of the smooth-halo annihilation luminosity.

Figure 6 shows the cumulative boost factor $B(<r)$ as a function of radius. In the top panel we plot curves for $\beta=$ $0,0.1,0.3,0.5,0.7$, and 1.0 , assuming no saturation. In the bottom panel we fix $\beta=1$ (except for the reference $\beta=0$ case) and vary the saturation velocity, $v_{\text {sat }} / c=10^{-4}$, $10^{-5}, 10^{-6}, 10^{-7}$, and 0 . Increasing $\beta$, or lowering $v_{\text {sat }}$ at a fixed $\beta$, leads to significant increases in the cumulative boost factor. For example, the total boost factor from within the virial radius of $\sim 300 \mathrm{kpc}$ grows from 17 ( $\beta=$ $0)$ to $1.3 \times 10^{6}$ for $\beta=1$ without saturation. Even with $v_{\text {sat }} / c=10^{-4}$, the $\beta=1$ case still results in about an order of magnitude increase in the total boost, to $\sim 120$.

\section{APPLICATION TO WIMP MODELS}

We now assemble estimates for the numerical values of $v_{0}$ and $\rho_{\max }$ for WIMP models. After freezeout of WIMP annihilation in the early Universe, the WIMPs may continue to scatter from the more abundant light standard model particles. These scatterings suppress perturbations in the WIMP density on subhorizon scales until these elastic-scattering interactions cease; i.e., at kinetic decoupling. This postfreezeout kinetic coupling of WIMPs suppresses primordial perturbations on mass scales smaller than $M_{c} \simeq 33\left(T_{\mathrm{kd}} / 10 \mathrm{MeV}\right)^{-3} M_{\oplus}[6]$, where $T_{\mathrm{kd}}$ is the kinetic-decoupling temperature. The smallest substructures in the Milky Way halo will therefore have formation masses no smaller than $M_{c}$. Close to the halo center, tidal interactions and impulsive stellar encounters may remove some fraction of this mass [12], but the dense cuspy cores contributing the majority of the annihilation luminosity likely survive [13]. Detailed calculations of the relevant elastic-scattering reactions shows that in supersymmetry and universal extra dimension models for WIMPs, this mass scale spans the range $10^{-6} M_{\oplus} \lesssim M_{c} \lesssim 100 M_{\oplus}$ [11], the precise value depending on the particle-physics details. Objects in this mass range undergo gravitational collapse at a redshift $z_{c} \simeq 40-\log _{10}\left(M_{c} / M_{\oplus}\right)$ [20]; the weak dependence of the collapse redshift on $M_{c}$ arises from the $n \rightarrow-3$ limit of the primordial power spectrum at small scales. These first collapsed objects obtain virial velocities $v_{\text {vir }}=v_{0} \simeq 1.0 \times 10^{-3}\left(M_{c} / M_{\oplus}\right)^{1 / 3} \times$ $\left(z_{c} / 40\right)^{1 / 2} \mathrm{~km} \mathrm{~s}^{-1}$ and virial densities $\rho_{\mathrm{vir}} \simeq$ $16\left(z_{c} / 40\right)^{3} \mathrm{GeV} \mathrm{cm}^{-3}$, corresponding to $\rho_{\max }=$ $80\left(z_{c} / 40\right)^{3}(c / 3.5)^{3} f(3.5) / f(c) \mathrm{GeV} \mathrm{cm}^{-3}$.
For a given WIMP model, the cutoff mass $M_{c}$ can be calculated and thus $\rho_{\max }$ and $v_{0}$ obtained. Given these parameters, our Eq. (10) can provide the boost factor, as a function of radius, for given Sommerfeld parameters $\beta$ and $v_{\text {sat }}$.

For example, suppose we would like a substructure boost of $B(r) \simeq 10^{3}$ locally to account for reported cosmic-ray anomalies. For an Earth mass cutoff $\left(M_{c}=M_{\oplus}, v_{0}=\right.$ $\left.1.0 \times 10^{-3} \mathrm{~km} \mathrm{~s}^{-1}, \rho_{\max }=80 \mathrm{GeV} \mathrm{cm}^{-3}\right)$, taking $\alpha=$ 1 , and a Sommerfeld model without saturation, the boost factor in Eq. (10) becomes

$$
B(r)=\frac{\left(1-f_{s}\right)}{1+1.75 \beta} \frac{\rho_{\max }}{\bar{\rho}}\left(\frac{v_{\mathrm{MW}}}{v_{0}}\right)^{\beta},
$$

which is $\gtrsim 10^{3}$ for $\beta \gtrsim 0.69$. Likewise, assuming $\beta=1$, a saturation velocity $v_{\text {sat }} / c \lesssim 3.3 \times 10^{-7}$ is necessary to get a local substructure boost factor greater than $10^{3}$.

\section{ANGULAR DEPENDENCE OF THE GAMMA-RAY INTENSITY}

We now consider the dependence of the intensity (photons $\mathrm{cm}^{-2} \mathrm{~s}^{-1} \mathrm{sr}^{-1}$ ) of gamma-ray radiation from WIMP annihilation in the Milky Way halo as a function of the angular separation $\psi$ between a given line of sight and the Galactic center. This intensity $I(\psi)$ can be written as an

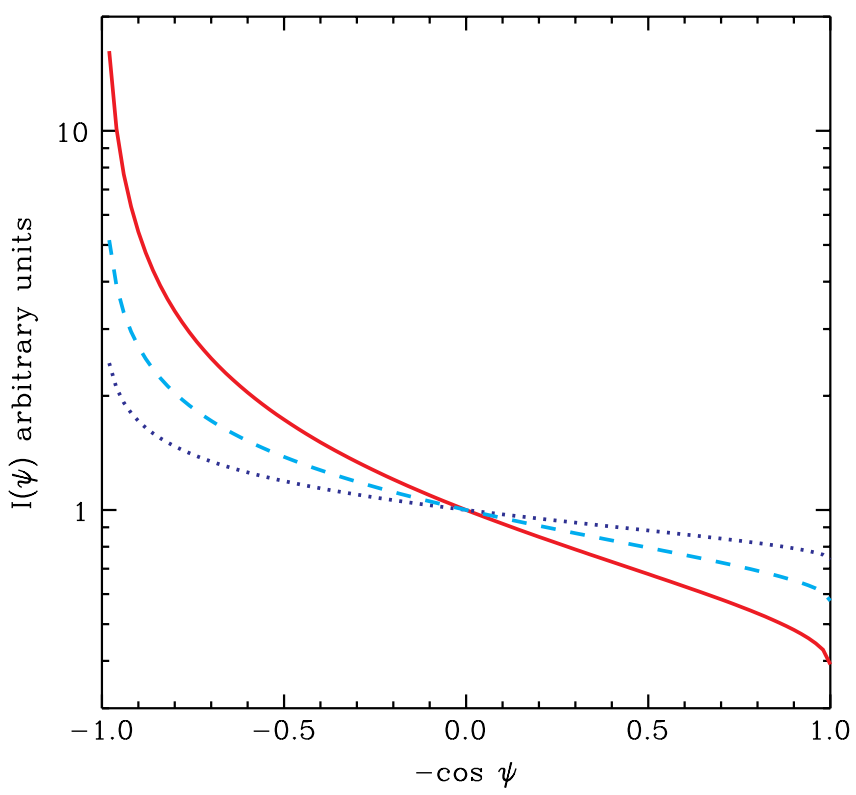

FIG. 7 (color online). Intensity of gamma-ray radiation from WIMP annihilation in the Milky Way as a function of the (cosine of the) angle $\psi$ that the line of sight makes with the Galactic center. All three curves are normalized to have the same intensity at $\cos \psi=0$. The top (solid) curve is the intensity due to annihilation in a smooth halo, i.e., for $I \propto \int \rho^{2} d l$. The bottom (dotted) curve is that for annihilation entirely in subhalos, i.e., $I \propto \int \rho d l$. The middle (dashed) curve is for a Sommerfeldenhanced annihilation [i.e., Eq. (11)] with $\beta=1, v_{\text {sat }} / c=$ $5.0 \times 10^{-5}$. 
integral

$$
\begin{aligned}
I(\psi) \propto & \int_{0}^{\infty} d l\left[\bar{\rho}\left(\sqrt{l^{2}+R_{0}^{2}-2 l R_{0} \cos \psi}\right)\right]^{2} \\
& \times B\left(\sqrt{l^{2}+R_{0}^{2}-2 l R_{0} \cos \psi}\right),
\end{aligned}
$$

along a line-of-sight distance $l$. If the halo is smooth, then $B(r)=1$, and the integral is $I \propto \int \bar{\rho}^{2} d l$, of the square of the smooth-halo density along the line of sight. If all of the annihilation in the halo took place in highly dense and very small subclumps, then the intensity would depend on an integral $I \propto \int \bar{\rho} d l$ simply of the density (rather than density squared). If the intensity depends on the integral of $\bar{\rho}^{2}$, then the intensity will vary more rapidly with $\psi$, rising rapidly toward the Galactic center, than if it depends on the integral of $\bar{\rho}$, as shown in Fig. 7.

Most generally, there may be annihilation in both the smooth component and in the clumped component, in which case the angular dependence of the intensity will fall somewhere in between [21], as illustrated in Fig. 7. Measurement of this angular variation may help shed empirical light on the existence of a boost factor.

\section{DISCUSSION}

It has long been recognized that there may be a hierarchy of structure in the Milky Way halo, with substructure existing all the way down to the mass scale of a fraction of an Earth mass, more than 22 orders of magnitude from the $\sim 10^{12} M_{\odot}$ Milky Way halo mass. It has also been noted that this substructure may have serious implications for the dark-matter-annihilation rate in the halo. If the WIMP has the canonical velocity-independent $\langle\sigma v\rangle$, then the boost factor could be as high as $\left(\rho_{\max } / \bar{\rho}\right) \sim 200$, if all of the substructure was preserved. If the WIMP has a Sommerfeld enhancement to the annihilation rate, the boost factor could be even higher, and perhaps dramatically so.

Unfortunately, the 22 or more orders of magnitude between the substructure cutoff mass and the Milky Way mass prevent the survival of the smallest-scale substructure to be addressed directly with simulations, and reliable analytic calculations of the survival fraction are similarly difficult. In earlier work [15], we used the nearly selfsimilar behavior of hierarchical clustering to develop an analytic approach to estimate the substructure survival. The central result of that work was a prediction that $P(\rho)$, the probability of any given point in the Galactic halo having a local density $\rho$, will have a high-density power-law tail.

Here, we have fit the parameters of that analytic model to new $N$-body simulations that can resolve the highdensity tail in $P(\rho)$. The analytic model then allows us to extrapolate the behavior of the simulations to mass scales far below the simulation's resolution scale. As a result, we have a simple analytic expression for the distribution of dark-matter densities within the Milky Way halo, as a function of galactocentric radius. The key qualitative result is that the fraction of the Milky Way volume occupied by substructures is small $\left(1-f_{s} \lesssim 10^{-3}\right.$ in the central regions, $\sim 10^{-2}$ in the outskirts). In particular, when we calculate the boost factor for WIMPs with the canonical (i.e., no) dependence of $\langle\sigma v\rangle$ on the velocity, we find that it is small: only about $50 \%$ at the solar radius, and only $\sim 17$ for the total boost within the virial radius. The PDF, supplemented with a scaling for the subhalo velocity dispersion with subhalo density, allows us to also analytically estimate the boost factor from substructure in the presence of a Sommerfeld enhancement. The central result here is given in Eqs. (10) and (11), which provide a boost factor in terms of the power-law index $\beta$ for the velocity scaling of $\langle\sigma v\rangle$, the saturation velocity $v_{\text {sat }}$, the maximum substructure density $\rho_{\max } \simeq 80 \mathrm{GeV} \mathrm{cm}^{-3}$, and the velocity dispersion $v_{0}$ of the smallest halos. We estimate numerically that local ( $8 \mathrm{kpc}$ ) boost factors $\gtrsim 10^{3}$ can be obtained with $\beta \gtrsim$ 0.69 , or for $\beta=1$ with $v_{\text {sat }} / c<3.3 \times 10^{-7}$.

We also discussed the finite width of the density distribution smoothly distributed component of halo dark matter, but find that the effects of this finite width on the boost factor are small.

There are several caveats to our results and several improvements that can be pursued in future work. (1) Hierarchical clustering is not a precisely self-similar process. In particular, given that the primordial mass power spectrum $P(k) \rightarrow k^{n}$ has a power-law index that becomes smaller at higher $k$ (smaller distance/mass scales, earlier collapse times), the power-law index $\alpha$ in our work will probably have some scale dependence, becoming, if anything, larger at higher densities. Taking this into account, our estimates for the boost factors due to substructure are probably on the high side. (2) The small-scale mass cutoff $M_{c}$ for canonical WIMPs [11] may not apply if the new interactions required for a Sommerfeld enhancement are taken into account. The cutoff mass may therefore be very different. If it is much larger, than the boost factors will be reduced (substructures will not extend to such small scales). If the cutoff mass is much smaller, the boost factor may be increased relative to our estimates, but, given the weak dependence of $z_{c}$ and thus $\rho_{\max }$ on $M_{c}$, not by much. (3) While identification of the high-density power-law tail in $P(\rho)$ and measurement of its small amplitude constitute a big step forward, our $N$-body measurement of the parameters required to describe the distribution $P(\rho, r)$ can certainly be improved upon. It will be important in future work to measure $f_{s}(r)$ more precisely, and to measure and determine the $r$ dependence of $\alpha$ and $\Delta$. (4) Figure 3 indicates that at densities above $\rho_{\max }$, the $\alpha=0$ power law for $P(\rho)$ will steepen to something closer to $\alpha=2$. This steep high-density tail is due to the small- $r \rho \sim 1 / r$ dependence in the earliest NFW halos. For a canonical WIMP with a velocity-independent $\langle\sigma v\rangle$, this steepening 
will not affect the results, since the integrand in Eq. (6) is proportional to $\rho^{-\alpha}$ and hence dominated by the low density (i.e., near $\rho_{\max }$ ) end of this steep tail. With a Sommerfeld enhancement, however, the integrand is proportional to $\rho^{1.75 \beta-\alpha}$ [Eq. (9)], which for $\alpha=2$ is dominated by the high-density end if $\beta>8 / 7$. In other words, if $\beta>8 / 7$, the substructure annihilation enhancement will be dominated by the $1 / r$ cusps in the earliest NFW subhalos, rather than the $\alpha=0$ part of $P(\rho)$ that we have considered until now. A more detailed calculation of this effect would depend sensitively on the smallest radius at which the $1 / r$ NFW behavior is valid, and we leave such a study to future work.

\section{ACKNOWLEDGMENTS}

This work was initiated during a workshop, attended by all three authors, hosted by the Caltech/JPL Keck Institute for Space Studies. M. Kuhlen thanks the Theoretical Astrophysics Center at UC Berkeley for support and acknowledges the hospitality of KITP at UC Santa Barbara, where part of this work was completed. This work was supported at Caltech by DoE DE-FG03-92-ER40701 and the Gordon and Betty Moore Foundation, and in part by the National Science Foundation under Grant No. PHY0551164.
[1] G. Jungman, M. Kamionkowski, and K. Griest, Phys. Rep. 267, 195 (1996).

[2] D. Hooper and S. Profumo, Phys. Rep. 453, 29 (2007).

[3] D. P. Finkbeiner, Astrophys. J. 614, 186 (2004); G. Dobler and D. P. Finkbeiner, ibid. 680, 1222 (2008); D. Hooper, D. P. Finkbeiner, and G. Dobler, Phys. Rev. D 76, 083012 (2007); P. Jean et al., Astron. Astrophys. 407, L55 (2003); J. Knodlseder et al., ibid. 411, L457 (2003); G. Weidenspointner et al., in 5th INTEGRAL Workshop on the INTEGRAL Universe, edited by V. Schoenfelder, G. Lichti, and C. Winkler (ESA Publications, Noordwijk, The Netherlands, 2004), Vol. 552, p. 133; A. W. Strong et al., Astron. Astrophys. 444, 495 (2005); D. J. Thompson, D. L. Bertsch, and R.H. O'Neal, Jr., Astrophys. J. Suppl. Ser. 157, 324 (2005); O. Adriani et al. (PAMELA), Nature (London) 458, 607 (2009).

[4] J. F. Navarro, C. S. Frenk, and S. D. M. White, Astrophys. J. 490, 493 (1997).

[5] A. A. Klypin, S. Gottlober, and A. V. Kravtsov, Astrophys. J. 516, 530 (1999); S. Ghigna et al., Mon. Not. R. Astron. Soc. 300, 146 (1998); A. A. Klypin, A. V. Kravtsov, O. Valenzuela, and F. Prada, Astrophys. J. 522, 82 (1999); B. Moore et al., ibid. 524, L19 (1999); C. Boehm, P. Fayet, and R. Schaeer, Phys. Lett. B 518, 8 (2001); A. M. Green, S. Hofmann, and D. J. Schwarz, Mon. Not. R. Astron. Soc. 353, L23 (2004); J. Cosmol. Astropart. Phys. 08 (2005) 003; AIP Conf. Proc. 805, 431 (2005); E. Bertschinger, Phys. Rev. D 74, 063509 (2006); V. Berezinsky, V. Dokuchaev, and Y. Eroshenko, ibid. 77, 083519 (2008); J. Diemand, M. Kuhlen, and P. Madau, Astrophys. J. 667, 859 (2007).

[6] A. Loeb and M. Zaldarriaga, Phys. Rev. D 71, 103520 (2005).

[7] J. Diemand, B. Moore, and J. Stadel, Nature (London) 433, 389 (2005); L. Gao, S. D. M. White, A. Jenkins, C. Frenk, and V. Springel, Mon. Not. R. Astron. Soc. 363, 379 (2005); J. Diemand, M. Kuhlen, and P. Madau, Astrophys. J. 649, 1 (2006).

[8] D. Stiff, L. M. Widrow, and J. Frieman, Phys. Rev. D 64, 083516 (2001); K. Freese, P. Gondolo, and H. J. Newberg, ibid. 71, 043516 (2005); M. Zemp et al., Mon. Not. R.
Astron. Soc. 394, 641 (2009).

[9] L. Bergstrom, J. Edsjo, P. Gondolo, and P. Ullio, Phys. Rev. D 59, 043506 (1999); L. Bergstrom, J. Edsjo, and P. Ullio, ibid. 58, 083507 (1998); C. Calcaneo-Roldan and B. Moore, ibid. 62, 123005 (2000); A. Tasitsiomi and A. V. Olinto, ibid. 66, 083006 (2002); V. Berezinsky, V. Dokuchaev, and Y. Eroshenko, ibid. 68, 103003 (2003); A. Tasitsiomi, J. M. Siegal-Gaskins, and A. V. Olinto, New Astron. Rev. 48, 473 (2004); F. Stoehr, S. D. M. White, V. Springel, G. Tormen, and N. Yoshida, Mon. Not. R. Astron. Soc. 345, 1313 (2003); S. M. Koushiappas, A. R. Zentner, and T.P. Walker, Phys. Rev. D 69, 043501 (2004); E. A. Baltz, J.E. Taylor, and L.L. Wai, Astrophys. J. Lett. 659, L125 (2007); L. Pieri, E. Branchini, and S. Hofmann, Phys. Rev. Lett. 95, 211301 (2005); S. M. Koushiappas, ibid. 97, 191301 (2006); J. Diemand, M. Kuhlen, and P. Madau, Astrophys. J. 657, 262 (2007); V. Berezinsky, V. Dokuchaev, and Y. Eroshenko, J. Cosmol. Astropart. Phys. 07 (2007) 011; L. Pieri, G. Bertone, and E. Branchini, Mon. Not. R. Astron. Soc. 384, 1627 (2008); M. Kuhlen, J. Diemand, and P. Madau, Astrophys. J. 686, 262 (2008); B.E. Robertson and A.R. Zentner, Phys. Rev. D 79, 083525 (2009); M. Kuhlen, P. Madau, and J. Silk, Science 325, 970 (2009).

[10] X.-1. Chen, M. Kamionkowski, and X.-m. Zhang, Phys. Rev. D 64, 021302 (2001).

[11] S. Profumo, K. Sigurdson, and M. Kamionkowski, Phys. Rev. Lett. 97, 031301 (2006).

[12] H. Zhao, J. E. Taylor, J. Silk, and D. Hooper, Astrophys. J. 654, 697 (2007); H.-S. Zhao, J. Taylor, J. Silk, and D. Hooper, arXiv:astro-ph/0502049; V. Berezinsky, V. Dokuchaev, and Y. Eroshenko, Phys. Rev. D 73, 063504 (2006).

[13] T. Goerdt, O. Y. Gnedin, B. Moore, J. Diemand, and J. Stadel, Mon. Not. R. Astron. Soc. 375, 191 (2007).

[14] J. Hisano, S. Matsumoto, M. M. Nojiri, and O. Saito, Phys. Rev. D 71, 063528 (2005); K. Belotsky, Daniele Fargion, M. Khlopov, and R. V. Konoplich, Phys. At. Nucl. 71, 147 (2008; J. March-Russell, S. M. West, D. Cumberbatch, and D. Hooper, J. High Energy Phys. 07 (2008) 058; J.L. 
Feng, H. Tu, and H.-B. Yu, J. Cosmol. Astropart. Phys. 10 (2008) 043; J. L. Feng and J. Kumar, Phys. Rev. Lett. 101, 231301 (2008); N. Arkani-Hamed, D. P. Finkbeiner, T. R. Slatyer, and N. Weiner, Phys. Rev. D 79, 015014 (2009); M. Pospelov and A. Ritz, Phys. Lett. B 671, 391 (2009).

[15] M. Kamionkowski and S. M. Koushiappas, Phys. Rev. D 77, 103509 (2008).

[16] M. Vogelsberger et al., Mon. Not. R. Astron. Soc. 395, 797 (2009).
[17] N. Afshordi, R. Mohayaee, and E. Bertschinger, arXiv: 0911.0414.

[18] J. Diemand et al., Nature (London) 454, 735 (2008).

[19] V. Springel et al., Nature (London) 456, 73 (2008); P. Brun, T. Delahaye, J. Diemand, S. Profumo, and P. Salati, Phys. Rev. D 80, 035023 (2009).

[20] M. Kamionkowski and S. Profumo, Phys. Rev. Lett. 101, 261301 (2008).

[21] S. K. Lee, S. Ando, and M. Kamionkowski, J. Cosmol. Astropart. Phys. 07 (2009) 007. 